\title{
Acknowledgment to Reviewers of Education Sciences in 2020
}

\section{Education Sciences Editorial Office}

MDPI AG, St. Alban-Anlage 66, 4052 Basel, Switzerland

Peer review is the driving force of journal development, and reviewers are gatekeepers who ensure that Education Sciences maintains its standards for the high quality of its published papers. Thanks to the cooperation of our reviewers, in 2020, the median time to first decision was 15 days and the median time to publication was 37 days. The editors would like to express their sincere gratitude to the following reviewers for their precious time and dedication, regardless of whether the papers were finally published:

Abellán Hernández, María

Acton, Renae

Adam, Helen

Adusei-Asante, Kwadwo

Aenis, Thomas

Afitska, Oksana

Aguaded Gómez, José Ignacio

Aguaded-Gómez, José Ignacio

Aguaded-Ramírez, Eva

Aguado, Txetxu

Agudo Romeo, María Del Mar

Aguilera Morales, David

Ahn, Hyejeong Boyce

Aidonopoulou, Tereza

Akkari, Abdeljalil

Aláez, Marian

Al-Bahrani, Abdullah

Albarracín, Lluís

Albers, Peggy

Aleksandra, Rogowska

Aleksandrowicz-Pedich, Lucyna

Alemany Arrebola, Inmaculada

Alexandre, Marta Susana Filipe

Ali, Nadia

Alkaher, Iris

Alpay, Esat

Ambrósio, Susana

Amillano, Alejandro

Amos, Jennifer R.

Anastasakis, Marinos

Anastasiu, Livia

Anastasovitis, Eleftherios

Andrews, Jean

Anguita, Juana María

Anne Kastens, Kim
Antinienė, Dalia

Arias De Sanchez, Gabriela

Arias-Correa, Azucena

Arnseth, Hans Christian

Arora, Amandeep

Atanasova, Tatiana

Aubry, Pascal

Babcock, Rebecca

Bacanin, Nebojsa

Bachiorri, Antonella

Badau, Adela

Badulescu, Alina

Bai, Li

Bailén, Amparo Huertas

Ballam, Nadine

Balli, Faruk

Banfi, Chiara

Barbosa, Ana

Barbosa, Gabriela

Barker, John

Barragán Martín, Ana Belén

Barragán-Sánchez, Raquel

Barrios-Garrido, Héctor

Bartz, Janieta

Basic, Goran

Basik, Sergei

Baskonus, Haci Mehmet

Bay, Jacquie L.

Bayram-Jacobs, Durdane

Beames, Simon

Beard, Larry

Beck, Moriah

Belda, José

Belford, Nish

Belmonte, Jesús López 
Benedek, András

Benson, Carolyn

Bergersen, Ane

Bernadowski, Carianne

Bernal-Bravo, César

Bernauer, James A.

Bernier, Andrew

Beyerlein, Steven

Bianco, Rosella

Biddinika, Muhammad Kunta

Bilancini, Ennio

Bjorklund, Peter

Blasco, Fernando

Blaško, Rudolf

Bobkina, Jelena

Bocsi, Veronika

Boerchi, Diego

Bögenhold, Dieter

Boldermo, Sidsel

Bolli, Thomas

Bollig, Georg

Bolondi, Giorgio

Bores García, Daniel

Borges, Africa

Borzikowsky, Christoph

Bosworth, Steven

Botham, Paola

Bowker, Cheryl L.

Bowlick, Forrest

Božič, Borut

Brady, Michael P.

Brajčić, Marija

Breeze, Ruth

Brennan Grieve, Victoria Luna

Bresciani, Sabrina

Breunig, Mary

Brewe, Eric

Brinia, Vasiliki

Broadhead, Samantha

Bronikowski, Michał

Brooks, Chay

Brosnan, Kevin

Brown, Gradyne

Brown, Lori J.

Brownell, Cassie

Brunderman, Lynnette

Bruni, Robert

Buckley, Jeffrey

Bugaj, Justyna Maria

Buntins, Katja

Bürki, Yvette
Burlacu, Sorin

Burlea-Schiopoiu, Adriana

Burrows, Andrea

Burrows, Andrea C.

Buschmann, Jürgen

Büssing, Alexander Georg

Butcher, John

Buzón-García, Olga

Buzzetto-Hollywood, Nicole A.

Bylieva, Daria

Byun, Chung Gyu

Cáceres Reche, Pilar

Caggiano, Valeria

Cahill, Helen

Caldeiro Pedreira, Mari Carmen

Callahan, Carolyn M.

Camarero-Figuerola, Marta

Campbell, Chris

Campbell, Janine

Cañabate, Dolors

Cannas Da Silva, Luisa

Cao, Ying

Căpușneanu, Sorinel

Carcedo González, Rodrigo J.

Carmona, Pedro

Caroline, Caroline

Carrera, Carlos Carbonell

Carrión-Martínez, José Juan

Carter, Amanda VandeHei

Cassidy, Claire

Castaldi, Elisa

Castañeda, Linda

Castillo-Rodríguez, Alfonso

Cavia Naya, Victoria

Cekić-Jovanović, Olivera

Centurino, Victoria A.S.

Cerbin-Koczorowska, Magdalena

Cerna, Miloslava

Cervera-Garvi, Pablo

Cervi, Laura

Cevasco, Roberta

Cha, Jeongho

Chabay, Ilan

Chan, Hsun-Yu

Chandrasekaran, Siva

Chang, Cheng-Chieh

Chang, Chi-Cheng

Chao Fernandez, Rocio

Chen, Chao-Chen

Chen, Liang-Bi

Chen, Liwen 
Chen, Whai-En

Cheng, Albert

Chien, Yu-Hung

Chiew Hong, Ng

Chistolini, Sandra

Chiu, An An

Chiu, Po-Sheng

Cho, Hye Jung

Cho, Jeong-Hyung

Chou, Pao-Nan

Chowdhury, TBM

Choy, Sarojni

Chrisman, Matthew

Cimermanová, Ivana

Činčera, Jan

Cinganotto, Letizia

Ciumara, Tudor

Clarke, Linda

Cobb, Casey

Cochran, Geraldine

Codella, Roberto

Coelho, Antonio

Coker, David

Collin, Kaija

Colomer Feliu, Jordi

Coman, Claudiu

Comber, Barbara

Concina, Eleonora

Conejero, Angela

Consorti, Fabrizio

Cooper, John B.

Coppi, Antonella

Corrales-Serrano, Mario

Coulter, Maura

Crawford, Joseph

Creely, Edwin

Crisol-Moya, Emilio

Crisp, Geoffrey

Crockett, Jean

Cropley, David

Cruise, Edie

Cruz, Orlanda

Cucuzzella, Carmela

Cutajar, Maria

Dache, Amalia Z.

Daddow, Angela

Dalby, Diane

Dale, Vicki

Daly, Nicola

Damiani, Paola

Daniela, Linda
Daradoumis, Thanasis

Davies, Randall S.

Davila-Velderrain, Jose

Dawson, Ross

De Barros Camargo, Claudia

De Chambrier, Anne-Françoise

De Craemer, Marieke

De Graaff, Erik

De La Fuente-Solana, Emilia I.

De La Rosa, Antonio Luque

De La Rosa, Olga María Alegre

De Pace, Francesco

De Souza, Claudia Daniele

De Troyer, Olga

Del Cerro Velázquez, Francisco

Delgado Valdivieso, Karina

Delgado, Gozalo

Delgado-Algarra, Emilio José

Delgado-Martín, Laura

Demack, Sean

Dempsey, Heidi L.

Depaepe, Fien

Dieckmann, Jack A.

Díez-Bedmar, María Del Consuelo

Dima, Gabriel

Dimitrova, Elena

Diogo, Sara

Domalewska, Dorota

Dommett, Eleanor

Dóniz-Páez, Javier

Dooley, Kim

Dos Reis, Pedro Guilherme Rocha

Dos Santos, Luis Miguel

Dougherty, Kevin

Doumanis, Ioannis

Drăgoi, Mihaela Cristina

Drasgow, Erik

$\mathrm{Du}$, Bowen

Dubrowski, Adam

Duffield, Mark

Duffin, Michael

Dumitras, Diana

Dumitriu, Dan

Duradoni, Mirko

Duranczyk, Irene

Duzhin, Fedor

Dziechciarz, Józef

Dziechciarz, Marta

Ealy, Julie

Edirisingha, Palitha

Eichengreen, Adva 
Eilks, Ingo

Elasra, Amira

Elgendy, Mostafa

Elia, Gianluca

Eliassi, Barzoo

Eradze, Maka

Erhabor, Edobor Michael

Eriksson, Urban

Escudero, David Fonseca

Espino-Díaz, Luis

Espoz-Lazo, Sebastián

Estellés, Marta

Everitt, Julia

Fang, Wei-Ta

Farajpour, Ali

Farber, Matthew

Farmer, Lesley

Farrar, Jennifer

Feltrero, Roberto

Felzmann, Dirk

Fernandez-Ahumada, Elvira

Fernández-Arias, Pablo

Fernández-Berkes, Eva

Fernández-Martín, Francisco Domingo

Fernández-Martínez, María Del Mar

Fernández-Salinero, Samuel

Ferrari, Enzo

Ferreira, Maria Eduarda

Feser, Markus

Fidan, Ismail

Filipe, José Antonio

Fiore, Eleonora

Fischer, Jean-Paul

Fischetti, John

Fondren, Kristi M.

Fortus, David

Foucrier, Tamsin

Fragou, Olga

Francis, Jacqueline

Freeman, Emily

Friesen, Sharon

Frondizi, Rocco

Fucà, Romina

Fuentes Cabrera, Arturo

Gabaldon-Estevan, Daniel

Gajek, Elżbieta

Galatanu, Olga

Gallardo Vázquez, Dolores

Gamble, Jeffrey Hugh

Gamo, Javier

Gao, Fei
Gao, Xuesong (Andy)

García Cuenca, Laura

García, Fernando

García, Jesús Manuel Nieto

Garcia, Jose Manuel

García-Garnica, Marina

García-Gómez, Andrés

García-Martín, Sheila

García-Martínez, Inmaculada

García-Milà, Mercé

García-Pérez, Lía

García-Ruiz, Carmen Rosa

Garman, Anthea

Gatt, Suzanne

Gavilán Izquierdo, José María

Gawel, Aleksandra

Gawlik-Kobylińska, Małgorzata

Gayoso-Cabada, Joaquín

Geelan, David

Georgiadou, Elissavet Gina

Georgiou, Helen

Gewerc, Adriana

Gianfredi, Vincenza

Giannakas, Filippos

Gibson, David

Gibson, Simone

Gibson, Suanne

Gierczyk, Marcin

Gifford, Alison

Gil Madrona, Pedro

Gillen, Julia

Gillies, Robyn

Giovanni, Vincenzi

Girbacia, Florin

Glass, Karen C.

Golcarenarenji, Gelayol

Gómez Carrasco, Cosme Jesús

Gómez Galán, José

Gómez García, Gerardo

Gómez Lacabex, Esther

Gomez Lopez, Raquel

Gómez-García, Melchor

Gómez-Urquiza, Jose L.

Gonçalves, Vítor

González, Daniel

González-Falcón, Inmaculada

González-Gómez, David

Gonzalez-Gonzalez, Hugo

Gora, Ana Alexandra

Gorard, Stephen

Gordon, Neil 
Goschin, Zizi

Grabinski, Konrad

Graham, Tammy J.

Grande-de-Prado, Mario

Gray, Donald

Greenough, Christopher

Gregori, Pablo

Gregson, Maggie

Gregurović, Margareta

Grimalt Álvaro, Carme

Grivokostopoulou, Foteini

Grospietsch, Finja

Grosseck, Gabriela

Guevara, Jennifer

Gustafsson, Peter

Gusy, Burkhard

Hadgraft, Roger

Halper, Leah

Hamed, Moussa Boumadan

Hardy, Keiran

Harib, Khalifa H.

Harrison, David

Hartanto, Andree

Hartley, Kendall

Hartman, Maria C.

Hasebrook, Joachim

Hassan, Abeer

Hassinger-Das, Brenna

Hay, Ian

Hay, Peta

Hayes, Amanda

Hayes, Sonya D.

Heikkilä, Mia

Heinicke, Xaver

Henriques, Paulo L.

Heo, Jinmoo

Herd, Colin

Hergueta Covacho, Elisa

Hermon, Sorin

Hernández Carrera, Rafael Manuel

Hernandez De Polaczyk, Ana

Hernández, Antonio Fernández

Hernández, Marta

Hernández-Valencia, Miguel

Hernando Lloréns, Belén

Herranen, Jaana

Herrera-Peco, Iván

Herrero Cosío, Álvaro

Hertzog, Nancy

Hidalgo, Nina

Higueras-rodríguez, lina
Hinojo Lucena, Francisco Javier

Hite, Rebecca

Hitka, Miloš

Hojan, Katarzyna

Holbrook, Jack

Hoople, Gordon

Horell, Harold

Hornby, Garry

HOW, Meng-Leong

$\mathrm{Hu}$, Shiow-Lin

Hubáčková, Šárka

Hubálovský, Štěpán

Huertas Abril, Cristina A.

Huhmarniemi, Maria

Huijser, Henk

Hunt, Jodi

Husband, Gary

Hussain, Sajjad

Hutchison, Laveria

Huxtable-Thomas, Louisa

Ianos, Maria Gratiela

Ihle, Andreas

Indiana, Maria Luisa

Indorf, Jane

Infante-Moro, Juan Carlos

Inglés, Cándido

Iñigo, Victoria

Inmaculada Diaz, Aznar

Iordache-Platis, Magdalena

Irizarry, Jose

Issa, Tomayess

Jagielska, Katarzyna

Jahodova Berkova, Andrea

Jáki, Erika

Jančaříková, Kateřina

Janikowska, Grażyna

Janko, Eleni

Jansen, Lisa $\mathrm{T}$.

Jean-Rémi, Lapaire

Jensen, Jamie L.

Jevtic, Adrijana Visnjic

Jia, Yunyan Andrea

Jiménez-Eguizabal, Juan Alfredo

Johnson, John

Johnston, Linda

Joosten-ten Brinke, Desirée

Jukic, Tonca

Jungbauer-Gans, Monika

Jurado De Los Santos, Pedro

Kacetl, Jaroslav

Kajamies, Anu 
Kaletova, Tatiana

Kalogiannakis, Michail

Kans, Mirka

Kapelari, Suzanne

Karakašić, Mirko

Karalekas, Georgios

Karayiannis, Nikos

Kärkkäinen, Sirpa

Karlos, Stamatis

Karpacz, Jarosław

Kashirsky, Dmitry

Kaur, Berinderjeet

Kawala-Sterniuk, Aleksandra

Kaya, Volkan Hasan

Keinonen, Tuula

Kelsall, Chris

Kervin, Lisa

Khan, Dawar

Kicinski, Marcin

Kilbrink, Nina

Kilty, Trina J.

Kimanen, Anuleena

Kinash, Shelley

Kinchin, Ian

Kinser, Kevin

Kioupi, Vasiliki

Kirkham, Sandra

Kisovar Ivanda, Tamara

Klichowski, Michal

Klímová, Blanka

Ko, Hsin-Chi

Kobus, Artur

Koh, Elizabeth

Koh, Kim

Kokić, Ivana Batarelo

Kokkonen, Tommi

Kolivand, Hoshamg

Kolvoord, Bob

Komotar, Maruša Hauptman

Koole, Marguerite

Koponen, Ismo

Kopylov, Yuri

Koster, Andries

Kostoulas-Makrakis, Nelly

Kouis, Dimitris

Kozhevnikov, Mikhail

Kralova, Zdena

Kreider, Consuelo M.

Kreja, Ireneusz

Krejcar, Ondrej

Krell, Moritz
Kroese, Floor

Kubsch, Marcus

Kubsch, Markus

Kuo, Shu-lung

Kupiainen, Reijo

Kuusisto, Arniika

Kuzmanovic, Marija

Laanpere, Mart

Laari-Salmela, Sari

Labastida, Ignasi

Lacy, Sara

Lai, Hollis

Lancaster, Simon J.

Lantz, Brendan

Lara Sánchez, Amador Jesús

Larsen, Thomas

Larsson, Johan

Lau, Jesslyn

Laurisz, Norbert

Law, Ka Ho

Leão, Celina

Leclercq, Virginie

Lee, Chang Won

Lee, Chia-Cheng

Leiva Olivencia, Juan J.

León, Samuel P.

Lepkova, Natalija

Lesterhuis, Marije

Lewis, Kathryn

Lhotska, Lenka

Li, Jingya

Lim, JongBeom

Lima, Rui M

Limbrick, Libby

Lin, Tyrone $\mathrm{T}$.

Linares, José Jesús Gázquez

Lio, Yuhlong

Lioukas, Spyros

Lis, Andrzej

Little, Chris

Llorente-Cejudo, Carmen

Lodi, Ernesto

Lonkwic, Paweł

Lopes, Betina Silva

López Díaz, Alfonso Isidro

López Rodríguez, Marta Sofía

López, José Luis

Lopez, Stella

López-Chao, Vicente

Lorca Marin, Antonio Alejandro

Lorenzo Lledó, Alejandro 
Lovyagin, Yuri N.

Lozano, Adele

Łubianka, Beata

Lucas, Margarida

Luis Ubago, Jose

Łukasik, Joanna

Lygo-Baker, Simon

Lyons, Roisin

Macdonald, Marey Allyson

Macedo, João Carlos

Mackinnon, Allan

Magli, Francesca

Magrane, Diane

Magreñán, Ángel Alberto

Mahan, Michael

Maier, Veronica

Makrakis, Vassilis

Malandrakis, George

Mamen, Asgeir

Mamlok-Naaman, Rachel

Manasia, Loredana

Manca, Stefania

Manco, Altay

Maněnová, Martina

Manero, Albert

Maniou, Theodora

Maraffi, Sabina

Marappan, Raja

Marbán, José María

Marcos-Merino, José María

Margherita, Alessandro

Marin, Victori

Marinaş, Laura Elena

Marín-Díaz, Verónica

Marinello, Francesco

Marín-Marín, José-Antonio

Marqués Molías, Luis

Marques, Arcelina

Martín Leralta, Susana

Martin, Jennifer

Martín, Jesús Sánchez

Martínez García, Vicente

Martínez Ramón, Juan Pedro

Martinez Ruiz, Maria Angeles

Martínez Sanahuja, Silvia

Martínez-Fernández, María Cristina

Martínez-León, Natalia

Martins, Fernando M. L.

Martins-Loução, Maria Amélia

Mason, Jon

Matarese, Valerie A.
Mataruna-Dos-Santos, Leonardo Jose

Matosas-López, Luis

Matusch, Tobias

Maurer, Patric

Mavragani, Amaryllis

Maya, Jesús

Mayoral, Asunción M.

Mazur, Joanna

McAdam, Julie

McAlister-Shields, Leah

McCuen, Richard

McCullough, Laura

Mckendry, Stephanie

McMeeking, Laura B. Sample

Medová, Janka

Meerts-Brandsma, Lisa

Meinel, Christoph

Melían-Díaz, Dámari

Melles, Gavin

Mena De Torres, Jaime

Méndez-Martínez, Carlos

Méndez-Suárez, Mariano

Meneses, Eloy López

Mesko, Maja

Métioui, Abdeljalil

Meyer, Helen

Meyer, Jonas

Miguez-Salinas, Gabriela

Milani, Alfredo

Milichovský, František

Milton, Marion

Miñana-Signes, Vicente

Miñano Rubio, Rafael

Mîndrescu, Veronica

Minier, Marta

Miotto, Giorgia

Miranda, Guilhermina Lobato

Mireles-Rios, Rebeca

Mirete, Lucía

Mishchuk, Halyna

Miskolczi, Péter

Mitchell, Shamika Ann

Mitroiu, Simona

Molero Jurado, María Del Mar

Molero, David

Molina, Marta

Molina-Plaza, Silvia

Molnár, György

Moniuszko, Hanna

Montero-Carretero, Carlos

Montes, Alejandro 
Montoro, Miriam Agreda

Montusiewicz, Jerzy

Moodley, Raymond

Morais Marques, Margarida

Morales, María

Moreno-Rodriguez, Ricardo

Morgan, Hani

Morgan, Joseph

Morgan, T. Lee

Morgana, Valentina

Mortlock, Anita

Mosavi, Amir

Moseley, Alex

Motrico, Emma

Moudatsou, Maria

Mousavi, Amin

Moya Higueras, Jorge

Mukaetova-Ladinska, Elizabeta B.

Mulder-Nijkamp, Maaike

Müllner, Bernhard

Muresan, Gabriela Mihaela

Murphy, Phillip J.

Myszkowska-Ryciak, Joanna

Napal, María

Naranjo-Zeledón, Luis

Naray-Davey, Szilvia

Nascimbeni, Fabio

Navarro Pérez, José Javier

Neagu, Gabriela

Nemec, Radek

Nesbitt, Danielle

Neubauer, Deane

Nguyen, Thao Phuong

Nichols, William

Nickel, Anne-Marie

Nicolae, Mariana

Nicolau, Cristina

Nicolescu, Luminita

Nikolopoulou, Kleopatra

Nitkiewicz, Tomasz

Nobanee, Haitham

Norel, Mariana

Norsworthy, Beverley

North, Max M.

Norton, Frances

Norwich, Brahm

Nousiainen, Maija

Novas, Jorge Casas

Novikova, Irina

Novotný, Lukáš

O'Leary, Simon
Obeid, Nassim Saleh

Ochoa, Xavier

Odinokaya, Maria

Oganisjana, Karine

Oh, Inha

Olszewski, Abbie

Olusoga, Yinka

Orcos, Lara

Oropesa Ruiz, Nieves Fátima

Ortega-Sánchez, Delfín

Ortiz Revilla, Jairo

Osei, Joseph

Ossiannilsson, Ebba

Ovalle, María Antonia

Oyarbide-Zubillaga, Aitor

Paci, Chris

Paderewski, Patricia

Pagge, Jenny

Paige, David

Palacios-Rodríguez, Antonio

Pan, Yushan

Panitsides, Eugenia A.

Papachristou, Vicky

Papadakis, Stamatis

Papademetri-Kachrimani, Chrystalla

Papadopoulou, Agnes

Pardo-Garcia, Cristina

Paredes, Angel

Parejo Llanos, Jose Luis

Park, Kyae-Sung

Park, Soojung

Parra-González, María Elena

Parry, Catherine

Parv, Luminita

Patel, Saumil S.

Paul, Peter V.

Pedaste, Markus

Pelkey, Jamin R.

Pellas, Nikolaos

Peña, Marta

Perales Jarillo, Mikel

Perepa, Prithvi

Pérez-Brunicardi, Darío

Pérez-González, Carlos

Petrica, João

Petritoli, Enrico

Petrykowski, Piotr

Philbin, Simon

Piazza, Roberta

Piedade, João

Pikhart, Marcel 
Pin, Paolo

Piñero Charlo, José Carlos

Pintér, Ákos

Pinto, Manuel

Pipere, Anita

Pisarenko, Veronika

Pisoni, Galena

Pitetti, Ken

Plotz, Thomas

Poce, Antonella

Põldoja, Hans

Pompei, Alessandro

Ponticorvo, Michela

Popa, Daniela Popa

Poza-Vilches, Fátima

Pozo Sánchez, Santiago

Pozorski, Zbigniew

Probst, Lorenz

Przytuła, Sylwia

Puma, Paola

Queiruga Dios, Miguel Ángel

Quintana-Murci, Elena

Quqandi, Ebtehal

Rachwał, Tomasz

Raciti, Maria

Radescu, Radu

Radu, Cătălina

Raffaghelli, Juliana

Rahimi, Ebrahim

Ramachandran, Roshini

Ramanauskaite, Simona

Rambaud, Salvador Cruz

Ramkissoon, Haywantee

Ramos-López, Darío

Ramsey, John

Ran, Bing

Randall, Victoria

Rashid, Mizanur

Ratiu, Lucia

Rauschnabel, Phil

Rauschnabel, Philipp A.

Read, David

Rear, David

Redifer, Jenni

Redondo, Ignacio

Regueiro, Bibiana

Reid, Scott

Reis, Giuliano

Reis, João

Reis, Pedro

Reiss, Michael
Renta-Davids, Ana-Inés

Resch, Katharina

Reuter, Robert

Revuelta-Domínguez, Francisco-Ignacio

Reyes, Augustina

Reyhner, Jon

Ribera Puchades, Juan Miguel

Richardson, Alasdair

Riegel, Ulrich

Roberts, Grady

Robles-Gómez, Antonio

Roche, Mary Doyle

Rödel, Bodo

Rodgers, Kirsten

Rodrigo, Ma. Mercedes

Rodríguez Herrero, Pablo

Rodríguez, Cristóbal

Rodríguez, Francisco

Rodríguez, Susana

Rodriguez-Garcia, Antonio-Manuel

Rodríguez-Muñiz, Luis J.

Rogers, Chris

Rogers, Karen B.

Rojas-Cessa, Roberto

Romero Rodríguez, José María

Romero-Rodríguez, Luis Miguel

Rubio, Fernando

Rueda, Silvia

Ruipérez-Valiente, José A.

Ruiz Rodríguez, María Del Consuelo

Ruiz-Esteban, Cecilia

Ruiz-Palmero, Julio

Russell, Arlene A.

Rusu, Alina Simona

Rusu, Valentina Diana

Ruth, Alissa

Ryabov, Igor

Sáez López, José Manuel

Sagone, Elisabetta

Salaberri-Ramiro, María Sagrario

Sales, Dora

Salinas, Jesus

Salvador-García, Celina

Sampedro, Begoña Esther

Sanches Formosinho, Maria Das Dores

Sánchez Santamaría, José

Sanchez, Almudena

Sanchez-Perez, Maria Del Mar

Sánchez-Rodríguez, José

Sánchez-Zafra, María

Sandow, Shaun L. 
Sanna, Mäki

Santagati, Mariagrazia

Santos-Villalba, María Jesús

Sanz, Alejandro

Sarasa-Cabezuelo, Antonio

Sari, Dany Perwita

Saunders, Gillian N.

Scheuch, Martin

Schlosser, Kolson

Schmitz, Florian

Schulte, Kim

Scifo, Lidia

Scott, Jessica

Seah, Wee Tiong

Seeling, Patrick

Segura Robles, Adrian

Seidel, Sebastian

Servatius, Brigitte

Shaikh, Zaffar

Shang, Guowen

Shelton, Catharyn

Shintel, Hadas

Short, Kathy

Shuker, Mary Jane

Shwartz, Yael

Sienkiewicz-Małyjurek, Katarzyna

Sierra, Javier Ortuño

Silva, Anabela Martins

Silva, Rui

Sim, Kwong Nui

Simonson, Shawn R.

Sinakou, Eleni

Skidmore, James

Skvarciany, Viktorija

Slesarenko, Inga V.

Smyth, Emer

Snauwaert, Dale T.

Söbke, Heinrich

Sobrino-Garcia, Itziar

Sochor, Tomas

Söderman, Johan

Sofianidis, Angelos

Softic, Sandra Kucina

Solano Pinto, Natalia

Solar, María Leonor Conejeros

Soler Costa, Rebeca

Sonetti, Giulia

Sonwalkar, Nishikant (Nish)

Soto Varela, Roberto

Soto, Ana Torres

Soto-Varela, Roberto
Sousa, Maria José

Stagg Peterson, Shelley

Stankevičienė, Jelena

Stanny, Claudia J.

Stella, Massimo

Stern, Elsbeth

Sternberg, Robert J.

Stewart, Ian

Stewart, Wendy A.

Stinken-Rösner, Lisa

Stockman, Caroline

Stoian, Claudia

Stoica, Leonard

Stone, Heather Nicole

Stone, Kari L.

Strassman, Barbara

Strevens, Caroline

Styliaras, Georgios

$\mathrm{Su}$, Chung-Ho

Sudraba, Velga

Sujo-Montes, Laura

Sun, Hung

Sun, Peng

Sutherland, Margaret

Švaříček, Roman

Swacha, Jakub

Swail, Watson Scott

Szabo, Zsuzsanna

Szydło, Joanna

Tadesse, Tefera

Tadeu, Pedro

Taheri, Pooya

Talaghir, Laurentiu-Gabriel

Tan, Paul Juinn Bing

Tan, Sheng

Tang, Shifang

Tararova, Olga

Tarkowski, Maciej

Tarraga-Minguez, Raul

Taysum, Alison

Theilmann, Florian

Thodelius, Charlotta

Thomas, Minnie

Thurn, Christian Maximilian

Tian, Jing

Tick, Andrea

Tikkanen, Jenni

Tomažič, Iztok

Tomczak, Michał

Tomczyk, Łukasz

Tomé, María 
Țoniș Bucea-Manea, Rocsana

Torkar, Gregor

Toronov, Vladislav

Torres Gordillo, Juan Jesús

Torres, Joana

Tran, Carolyn

Traxler, Adrienne

Troia, Gary

Trompf, Garry

Trujillo-Torres, Juan M.

Truța, Camelia

Trybek, Grzegorz

Tsantopoulos, Georgios

Tso, Wing Bo

Tumosa, Nina

Turki, Houcemeddine

Tysor, Deborah

Uebernickel, Falk

Urquidi-Martín, Ana C.

Urrea-Solano, Mayra

Urrutia, Manuel Leon

Vain, Ants

Vaiopoulou, Julie

Väisänen, Pertti

Valantinaitè, Ilona

Vale, Isabel

Valtteri Pesonen, Henri

Valverde-Berrocoso, Jesús

Van Gorp, Koen

Van Herwegen, Jo

Vanderburg, Michelle

Varma, Sashank

Varouchas, Emmanouil

Vasilevna, Borzova Elena

Vaz De Carvalho, Carlos

Vazquez, Elena Perez

Vega-Marcote, Pedro

Veglis, Andreas

Vergara, Diego

Ververidis, Dimitrios

Vidal-Raméntol, Salvador

Vieira, Rui

Villarreal, Brandilynn

Vinader-Segura, Raquel

Vinci, Concetto Paolo

Vodă, Ana Iolanda

Vogelaar, Bart

Voskoglou, Michael

Vranješević, Jelena

Wagner, Steffen

Wainwright, John
Wakeman, Shawnee

Walker, Marvin

Walker, Richard

Walker-Gleaves, Caroline

Walkington, Helen

Wang, Shiow-Luan

Wang, Zhuoying

Warrington, Kayleigh L.

Wątróbski, Jarosław

Weathers, Jamie

Weeks, Faith

Weisblat, Irina

Wescoatt, Ben

Whitehead, Dean

Whitney, Jean

Wigal, Cecelia

Wilhelm, Jennifer

Williams, Peter

Winings, Kathy

Winter, Jennie

Wiora, Józef

Wohlers, Lars

Wolbring, Gregor

Woltenberg, Leslie

Woodfield, Lorayne

Woolcock, Geoffrey

Woosnam, Kyle Maurice

Wroblewski, Angela

$\mathrm{Wu}$, Leon Yufeng

$\mathrm{Wu}$, Manfred Man-Fat

$\mathrm{Wu}$, Min Lun

$\mathrm{Wu}$, Stephen

Wynn, Martin

Wyver, Shirley

Xie, Jingrong

Yáñez, Dionisio F.

Yang, Ping

Yang, Yi

Yao, Xiangquan (James)

Yli-Panula, Eija

Yoon, Susie

Zadra, Cinzia

Zajda, Joseph

Zamora-Polo, Francisco

Zapatera Llinares, Alberto

Zarobell, John

Zhang, Jize

Zhao, Shuo

Zheng, Lin

Ziese, Michael

Zion, Michal 
Zou, Ping

Zuccoli, Franca

Zukow, Walery
Żukowska, Hab. Joanna

Zurita Ortega, Félix 